\title{
The Edge Connectivity of Circuit Graphs of Matroids ${ }^{\star}$
}

\author{
Ping $\mathrm{Li}^{\star \star}$ and Guizhen Liu \\ Department of Mathematics, Shandong University, Jinan 250100, P.R. China \\ liping@math.sdu.edu.cn
}

\begin{abstract}
Let $G$ be the circuit graph of any connected matroid and let $\kappa^{\prime}(G)$ and $\delta(G)$ be the edge connectivity and minimum degree of $G$. It is proved that $\kappa^{\prime}(G)=\delta(G)$.
\end{abstract}

Keywords: Matroid, circuit graph of matroid, edge connectivity.

\section{Introduction}

We shall assume familiarity with graph theory and matroid theory. For terms that are not defined in this note, see 2 for graphs, and 8 for matroids. Let $\mathscr{C}$ be the family of circuits of a matroid $M$. It satisfies the following two axioms:

(C1) A proper subset of a member of $\mathscr{C}$ is not a member of $\mathscr{C}$.

(C2) If $a \in C_{1} \cap C_{2}$ and $b \in C_{1}-C_{2}$ where $C_{1}, C_{2} \in \mathscr{C}$ and $a, b \in E$, then there exists a $C_{3} \in \mathscr{C}$ such that $b \in C_{3} \subseteq\left(C_{1} \cup C_{2}\right)-\{a\}$.

Now we give a new concept as follows. The circuit graph of a matroid $M$ is a graph $G=G(M)$ with vertex set $V(G)$ and edge set $E(G)$ such that $V(G)=\mathscr{C}$ and $E(G)=\left\{C C^{\prime}\left|C, C^{\prime} \in \mathscr{C},\right| C \cap C^{\prime} \mid \neq 0\right\}$, where the same notation is used for the vertices of $G$ and the circuits of $M$.

Maurer discussed the relationship of bases of matroids and graphs and defined the base graph of a matroid 6, 77. Liu studied the connectivities of base graph of matroids [3,4]. Other related results can be found in [1,5]. In this paper we study the edge connectivity in the circuit graphs of matroids.

In this paper we will prove that the edge connectivity of the circuit graph of any connected matroid is equal to its minimum degree $\delta(G)$.

\section{Preliminary Results}

Lemma 1 [8]. A matroid $M$ is connected if and only if for every pair $e_{1}, e_{2}$ of distinct elements of $E$, there is a circuit containing both $e_{1}$ and $e_{2}$.

Lemma 2 [8]. If $M$ is a connected matroid, then for every $e \in E$, either $M / e$ or $M \backslash e$ is also connected.

\footnotetext{
* This research is supported by NSFC(60673047) and RSDP(20040422004) of China.

** Corresponding author.
} 
Lemma 3 [8]. Let $C$ and $C^{\star}$ be any circuit and co-circuit of a matroid $M$. Then $\left|C \cap C^{\star}\right| \neq 1$.

Lemma 4 [8]. If $M$ is a matroid and if $X$ and $Y$ are two disjoint sets of elements of $M$, then $(M \backslash X) / Y=(M / Y) \backslash X$.

\section{Main Results}

A matroid $M$ is trivial if it has no circuits. In the following all matroids will be nontrivial.

Next we will discuss the properties of the matroid circuit graph. To prove the main results we firstly present the following lemmas which are clearly true.

Lemma 5. Let $M$ be any nontrivial matroid on $E$ and $e \in E$. If $G$ and $G_{1}$ are circuit graphs of $M$ and $M \backslash e$, respectively, then $G_{1}$ is a subgraph of $G$ induced by $V_{1}=V(G)-V_{2}$ where $V_{2}=\{C \mid C \in \mathscr{C}, e \in C\}$.

Obviously the subgraph $G_{2}$ of $G$ induced by $V_{2}$ is a complete graph. By Lemma 5. $G_{1}$ and $G_{2}$ are induced subgraphs of $G$ and $V\left(G_{1}\right)$ and $V\left(G_{2}\right)$ partition $V(G)$.

Lemma 6. For any matroid $M=(E, \mathscr{C})$ which has a 2-cocircuit $\{a, b\}$, the circuit graph of $M$ is isomorphic to that of $M / a$.

Lemma 7. Suppose that $M=(E, \mathscr{C})$ is a connected matroid with two distinct circuits $C_{1}, C_{2}$ such that $\left|E-\left(C_{1} \cup C_{2}\right)\right| \geq 2$ and for any $e \in E-\left(C_{1} \cup C_{2}\right)$, $M \backslash e$ is disconnected. Then $M$ has a co-circuit of cardinality two.

Proof: We shall prove the theorem by induction on $\left|E-\left(C_{1} \cup C_{2}\right)\right|$. Suppose that $E-\left(C_{1} \cup C_{2}\right)=\left\{e_{1} \cup e_{2}\right\}$ and $M \backslash e_{i}(i=1,2)$ is disconnected. If $C_{1}$ and $C_{2}$ are in the same component of $M \backslash e_{1}$, and $\left\{e_{2}\right\}$ is the other component of $M \backslash e_{1}$, then any circuit containing $e_{2}$ contains $e_{1} .\left\{e_{1}, e_{2}\right\}$ is a co-circuit of $M$ because by (C2), if there is a circuit containing $e_{1}$ does not contain $e_{2}$ then there is a circuit containing $e_{2}$ does not contain $e_{1}$, which is a contradiction. If $C_{1}$ and $C_{2}$ are not in the same component of $M \backslash e_{1}$ and $e_{2}$ is in the same component with $C_{1}$, then $M \backslash e_{2}$ cannot be disconnected because there is a circuit containing $e_{1}$ intersects both $C_{1}$ and $C_{2}$ but does not contain $e_{2}$. Thus $\left\{e_{2}\right\}$ is another component of $M \backslash e_{1}$. Therefore $\left\{e_{1}, e_{2}\right\}$ is a co-circuit of $M$.

Suppose the result is true for $\left|E-\left(C_{1} \cup C_{2}\right)\right|=n-1$. We prove that the result is also true for $\left|E-\left(C_{1} \cup C_{2}\right)\right|=n>3$. Let $e_{1}$ be an element of $E-\left(C_{1} \cup C_{2}\right)$ and $M \backslash e_{1}$ is disconnected. Hence, by Lemma $2 . e_{1}$ is connected. Now if $e_{2}$ is any element of $E-\left(C_{1} \cup C_{2} \cup\left\{e_{1}\right\}\right)$, the matroid $\left(M \backslash e_{2}\right) / e_{1}$ is disconnected unless $\left\{e_{1}\right\}$ is a component of $M \backslash e_{2}$. If $\left\{e_{1}\right\}$ is a component of $M \backslash e_{2}$, then $\left\{e_{1}, e_{2}\right\}$ is a co-circuit of $M$. If $\left(M \backslash e_{2}\right) / e_{1}$ is disconnected for each $e_{2} \in E-\left(C_{1} \cup C_{2} \cup\left\{e_{1}\right\}\right)$, by Lemma 4. $\left(M / e_{1}\right) \backslash e_{2}=\left(M \backslash e_{2}\right) / e_{1}$, we have $\left(M / e_{1}\right) \backslash e_{2}$ is disconnected for all $e_{2} \in E-\left(C_{1} \cup C_{2} \cup\left\{e_{1}\right\}\right)$. By induction, $M / e_{1}$ has a co-circuit of cardinality two. A co-circuit of $M / e_{1}$ is also a co-circuit of $M$. We prove the Lemma.

Now we give the main theorem of this paper. 
Theorem 1. Suppose that $G=G(M)$ is the circuit graph of a connected nontrivial matroid $M=(E, \mathscr{C})$ and $C_{1}$ and $C_{2}$ are distinct vertices of $G$. Then $C_{1}$ and $C_{2}$ are connected by $d=\min \left\{d\left(C_{1}\right), d\left(C_{2}\right)\right\}$ edge-disjoint paths where $d\left(C_{1}\right)$ and $d\left(C_{2}\right)$ denote the degree of vertices $C_{1}$ and $C_{2}$ in $G$, respectively.

Proof: We shall prove the theorem by induction on $|E(M)|$. When $|E(M)|=3$, each element in $M$ is parallel to another. It is easy to see that $G(M)=K_{3}$. The theorem is clearly true. Suppose that the result is true for $|E(M)|=n-1$. We prove that the result is also true for $|E(M)|=n>3$. Let $C_{1}$ and $C_{2}$ be any two vertices in $G$.

There are two cases to distinguish.

Case 1: $\left(C_{1} \cup C_{2}\right)=E(M)$. It is easy to see that $C_{1}$ and $C_{2}$ are both adjacent to any circuit in $\mathscr{C}-\left\{C_{1} \cup C_{2}\right\}$ and the conclusion is obviously true.

Case 2: $\left(C_{1} \cup C_{2}\right) \neq E(M)$.

There are two subcases to distinguish.

Subcase 2.1: There is an element $e \in E(M)-\left(C_{1} \cup C_{2}\right)$ such that $M \backslash e$ is connected. Let $G_{1}=G(M \backslash e)$ be the circuit graph of $M \backslash e$ and $G_{2}$ be the subgraph of $G$ induced by $V_{2}$ where $V_{2}=\{C \mid C \in \mathscr{C}, e \in C\}$. Thus $C_{1}$ and $C_{2}$ are in $G_{1}$. By induction, in $G_{1}, C_{1}, C_{2}$ are connected by $d_{1}=\min \left\{d_{1}\left(C_{1}\right), d_{1}\left(C_{2}\right)\right\}$ edge-disjoint paths where $d_{1}\left(C_{1}\right)$ and $d_{1}\left(C_{2}\right)$ denote the degree of vertices $C_{1}$ and $C_{2}$ in $G_{1}$, respectively. Let $\mathscr{P}_{1}=\left\{P_{1}, P_{2}, \ldots, P_{d_{1}}\right\}$ be the family of shortest edge-disjoint paths connecting $C_{1}$ and $C_{2}$ in $G_{1}$. Without loss of generality, we may assume that $d_{1}\left(C_{1}\right) \geq d_{1}\left(C_{2}\right)$. There are two subcases to distinguish.

Subcase 2.1a: $d_{1}\left(C_{1}\right)=d_{1}\left(C_{2}\right)$. The conclusion is obvious because $G_{2}$ is a complete graph.

Subcase 2.1b: $d_{1}\left(C_{1}\right)>d_{1}\left(C_{2}\right)$. By induction, in $G_{1}$ there are $d_{1}=$ $\min \left\{d_{1}\left(C_{1}\right), d_{1}\left(C_{2}\right)\right\}=d_{1}\left(C_{2}\right)$ edge-disjoint paths connecting $C_{1}$ and $C_{2}$. Let $\mathscr{P}_{1}=\left\{P_{1}, P_{2}, \ldots, P_{d_{1}\left(C_{2}\right)}\right\}$ be the family of shortest edge-disjoint paths connecting $C_{1}$ and $C_{2}$ in $G_{1}$. It is obvious that each $P_{i}\left(i=1,2, \ldots, d_{1}\left(C_{2}\right)\right)$ contains exactly one vertex adjacent to $C_{1}$ and one vertex adjacent to $C_{2}$. Let $A_{1}, A_{2}, \ldots, A_{d_{1}\left(C_{1}\right)-d_{1}\left(C_{2}\right)}$ be the vertices in $G_{1}$ that are adjacent to $C_{1}$ but not contained in $d_{1}$ edge-disjoint paths. By Lemma 1 for any element $e^{\prime}$ in $A_{i}\left(i=1,2, \ldots, d_{1}\left(C_{1}\right)-d_{1}\left(C_{2}\right)\right)$ there is a circuit $A_{i}^{\prime}$ in $G_{2}$ containing $e$ and $e^{\prime}$, thus $A_{i} A_{i}^{\prime}$ is an edge in $G(M)$. Let $D_{1}, D_{2}, \ldots, D_{m}$ denote the vertices in $G_{2}$ that is adjacent to $C_{2} . G_{2}$ is a complete graph, so $A_{i}^{\prime}$ is adjacent to $D_{j}\left(i=1,2, \ldots, d_{1}\left(C_{1}\right)-d_{1}\left(C_{2}\right) ; j=1,2, \ldots, m\right)$. If $m \leq d_{1}\left(C_{1}\right)-d_{1}\left(C_{2}\right)$, $C_{1} A_{i} A_{i}^{\prime} D_{i} C_{2}$ are $m$ edge-disjoint paths connecting $C_{1}$ and $C_{2}$ where $A_{i}^{\prime}$ can be $D_{i}$ $(i=1,2, \ldots, m)$. Here it is possible that $A_{i}^{\prime}=A_{j}^{\prime}\left(i \neq j ; i, j=1,2, \ldots, d_{1}\left(C_{1}\right)-\right.$ $\left.d_{1}\left(C_{2}\right)\right)$. But it is forbidden that $D_{i}=D_{j}(i \neq j ; i, j=1,2, \ldots, m) \cdot d\left(C_{2}\right)=$ $d_{1}\left(C_{2}\right)+m \leq d_{1}\left(C_{1}\right)<d\left(C_{1}\right)$, thus $d=\min \left\{d\left(C_{1}\right), d\left(C_{2}\right)\right\}=d\left(C_{2}\right) . \mathscr{P}=\mathscr{P}_{1} \cup$ $\left\{C_{1} A_{1} A_{1}^{\prime} D_{1} C_{2}, C_{1} A_{2} A_{2}^{\prime} D_{2} C_{2}, \ldots, C_{1} A_{m} A_{m}^{\prime} D_{m} C_{2}\right\}$ are $d$ edge-disjoint paths connecting $C_{1}$ and $C_{2}$ in $G$ (see Fig.1).

If $m>d_{1}\left(C_{1}\right)-d_{1}\left(C_{2}\right)$, The proof is similar to that above. 


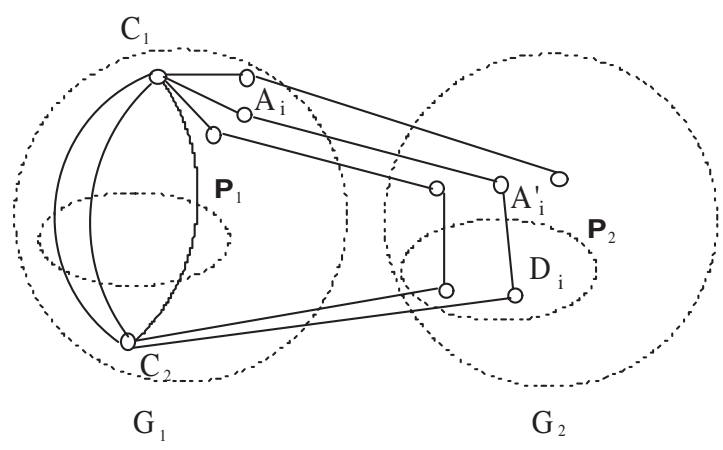

Fig. 1.

Subcase 2.2: There is no element $e \in E(M)-\left(C_{1} \cup C_{2}\right)$ such that $M \backslash e$ is connected. If $E(M)-\left(C_{1} \cup C_{2}\right)=\{e\}$ and $M \backslash e$ is disconnected, it is easy to see that $C_{1} \cap C_{2}=\emptyset$ and $C_{1}, C_{2}$ are the two components of $M \backslash e$. Thus any circuit of $M$ intersecting both $C_{1}$ and $C_{2}$ contains $e$, then $C_{1}$ and $C_{2}$ are both adjacent to any circuit in $\mathscr{C}-\left\{C_{1} \cup C_{2}\right\}$ and the conclusion is obviously true. Suppose that $\left|E(M)-\left(C_{1} \cup C_{2}\right)\right| \geq 2$ and for any $e \in E(M)-\left(C_{1} \cup C_{2}\right), M \backslash e$ is disconnected. By Lemma 7, $M$ has a 2-cocircuit $\{a, b\}$. By Lemma 6 the circuit graph of $M / a$ is isomorphic to that of $M$. By induction hypothesis, the result holds.

Thus the theorem follows by induction.

By Theorem 1 and Menger's Theorem, we can get the following corollary.

Corollary 2. Suppose that $G=G(M)$ is the circuit graph of a connected matroid $M$ with minimum degree $\delta(G)$. Then the edge connectivity $\kappa^{\prime}(G)=\delta(G)$.

\section{References}

1. Alspach, B., Liu, G.: Paths and Cycles in Matroid Base Graphs, Graphs and Combinatorics, 5(1989), 207-211

2. Bondy, J. A., Murty, U. S. R.: Graph Theory With Applications, Macmillan, New York, (1976)

3. Liu, G.: A Lower Bound on Connectivities of Matroid Base Graphs, Discrete Mathematics, 64(1988), 55-66

4. Liu, G.: The Proof of a Conjecture on Matroid Basis Graphs, Science in China, 6(A) (1990), 593-599

5. Liu, G., Zhang, L.: Forest Graphs of Graphs, Chinese Journal of Engineering Mathematics, 22(6)(2005),1100-1104

6. Maurer, S. B.: Matroid basis graphs 1, Journal of Combinatorial Theory Series B, 14(1973), 216-240

7. Maurer, S. B.: Matroid basis graphs 2, Journal of Combinatorial Theory Series B, 15(1973), 121-145

8. Oxley, J. G.: Matroid Theory, Oxford University Press, New York, 1992. 